\title{
Co-operation via conflicts in manufacturing systems
}

\author{
M. Horváth,
}

Technical University of Budapest, Egry J. u. 1, H-1111 Budapest, Hungary

Telephone: 36-1-4632515, Fax: 36-1-4633176

E-mail: h5893hor@ella.hu

\section{A. Márkus and J. Váncza}

Artificial Intelligence Laboratory, Computer and Automation Research Institute, Hungarian Academy of Sciences, P.O.B. 63, H-1518 Budapest, Hungary

Telephone: 36-1-2096194, Fax: 36-1-1667503

E-mail: \{markus;vancza\}@sztaki.hu

\begin{abstract}
The paper discusses how conflicts that emerge necessarily between various agents in a manufacturing system can be exploited in favour of the whole system. Experience related to conflict situations and their resolutions constitutes the core of the system's social knowledge. A mechanism for co-operative problem formulation and solution is suggested; this mechanism is based on the use of the recollections of previous conflict cases. As a vehicle for capturing this social knowledge and transferring it to the actual control of manufacturing systems, case-based reasoning is proposed, because it is able to handle incomplete, inconsistent and unformatted information, and can support incremental learning.
\end{abstract}

\section{Keywords}

Manufacturing, co-operating agents, conflicts, enterprise modelling 


\section{INTRODUCTION}

Conflicts inevitably happen time and again in any manufacturing system. Efficient operation, adaptability, and occasionally even the survival of a system hinges on whether its components are able to come out of conflict situations with a solution. The conflicts and their resolutions constitute the base of the so-called social knowledge - a kind of knowledge which emerges from the collective action of the individuals and, therefore, belongs to the system as a whole. We argue that instead of trying to eliminate conflicts (which is impossible), conflicts should be exploited in the control of manufacturing systems. However, this is possible only if we are able to capture and utilise the social knowledge embodied in the conflict resolution process of the organisation.

Our point is especially evident in a multi-agent setting where a manufacturing system can be modelled like an ensemble of relatively independent and selfinterested agents, each having goals directed toward their internal conduct and also toward the behaviour of the overall system. When striving to achieve their own goals and objectives, the agents get in conflict with each other; survival of the whole can be guaranteed only when they are able to continuously resolve their conflicts.

In the design and management of manufacturing systems, the problem of handling conflicts has put up a stout resistance to methods based on strict, wellformalised models and representations. In our opinion, the main trouble is that formal methods, if compared with human judgement, are just far too weak in distinguishing primary and secondary issues, weighting and re-evaluating options and preferences, giving similar answers to problems close to previous ones; in exploiting analogies and in remembering and forgetting. The goal of the paper is to show that just these capabilities can help us to seize the essence of conflicts and make use of this knowledge in the control of manufacturing systems.

\section{TOWARD MULTI-AGENT ORGANIZATION OF MANUFACTURING SYSTEMS}

Organisation structures, which bring information and decision rights together, can be considered as efficient responses to information costs. A change in information requirements and costs should be followed by a change in the organisational structure. When decisions are made relying more and more on local, situated and pragmatic knowledge the transfer of which across the organisation is rather costly and cumbersome, then the organisational structure should be changed accordingly: decision authority should be allocated to those who access the pertinent information and the knowledge of how to process it (Brynjolfsson and Mendelson, 1993). Co-locating decision rights and distributed information leads to a flatter management hierarchy and to a more decentralised control. This is what actually 
happens also in manufacturing systems when large, vertically integrated, hierarchical organisations are transformed into heterarchic, network organisations.

In general, there are three main factors that determine the structure of an organisation: (1) the incentive system, (2) the allocation of decision rights, and (3) the scheme for monitoring and measuring performance. Agents in a network organisation who are not - and can never be - completely aware of each others' goals and objectives have almost necessarily adverse incentives. Moreover, new information technologies allow them to handle more functions and to widen their span of interest and control. All these factors increase the potential of conflicts between the agents. Hence, a heterarchic organisation poses in itself severe limitations to its own efficient behaviour; its success depends on how well its agents can share information (communicate), co-operate and manage their work jointly.

It was long before manufacturing system theory suggested cautious and rather pessimistic organising principles for systems operating under turbulent market conditions. More relevant now than ever, these principles say that it is better to recognise ignorance than to presume knowledge; that it is better to regard the future unpredictable than to be prepared for expected events only (Hatvany, 1984, 1985). From this stance, there follows a heterarchic system structure with distributed responsibilities, tasks and resources (Dilts et al., 1991). Accordingly, manufacturing systems should be organised as loosely coupled networks of communicating and co-operating agents performing distinct tasks of their own (such as marketing, product design, manufacturing engineering, production control and scheduling, etc.). Redundant functions and responsibilities are necessary for absorbing sudden changes both in the internal and external conditions, especially if they may take unexpected forms. This hypothesis has been corroborated in part by practice: the success of some of the most competitive Japanese companies was attributed to the fact that their organisational structures had functional overlaps (Nonaka, 1991).

When taking the above approach, however, a further pessimistic assumption has to be accepted: that collaborative work and co-operation cannot automatically be guaranteed. In the multi-agent framework, the manufacturing game is played not by self-denying, altruistic and benevolent agents that interact through an institution that couples and aggregates their behaviour always in a predictable and optimal way.

\section{SOCIAL KNOWLEDGE AND CONFLICTS}

Recent results of organisational theory suggest that the human side of collaborative work is at least as important as the technical one. As it was pointed out, "the biggest roadblocks on the path to success are not the dynamic, complex, and even chaotic business environment or the evolving technology, but the people issues - 
the people dynamics" (Hyman, 1993). This position, extreme though it appears to be, harmonises with a recurrent idea that "in an economy where the only certainty is uncertainty, the one sure source of lasting competitive advantage is knowledge" (Nonaka, 1991). The most valuable asset of a factory or an enterprise is not its machines, equipment, infrastructure and materials, but the knowledge that keeps all this working and that reinvents itself whenever the need of adaptation dictates. Management, resource management, marketing, product design and development, sales, engineering, procurement and storage, production control and services has each its own, individual knowledge sources. Though, the factory's knowledge is not a simple conglomerate of these sources-it relates rather to the ways of using the specific task-related fragments in a concerted action so as to achieve both individual and overall goals.

There is a common understanding that members of any social organisation (groups, teams, companies, corporations) have some social knowledge (or corporate memory) that enables them to co-ordinate their actions and, more generally, to adapt their behaviour to changing conditions (see e.g., Minsky, 1985, Gasser, 1991, Nonaka, 1991, Constantine, 1993, Shaw and Fox, 1993). How does organisational cognition emerge from the collective action of individuals? What is the content of such socially constituted knowledge? Where is it located? It is senseless and useless in itself; it is good for nothing without the contribution of the agents' own, task-related individual knowledge.

Capturing social knowledge is by far not a matter of processing objective information. Rather, it needs tapping of hidden and often highly subjective insights, intuitions and hunches of individuals. In the multi-agent setting, so-called objective facts in and of themselves are clearly meaningless. Different agents define different contexts for the same facts through their actions and interactions. The data they use or report always assume some underlying interpretation (as Pirandello expressed, "a fact is like a sack: it will not stand up unless you put something in it"), the same phenomena may have several - often even contradictory - explanations. It must be accepted that the personal aspects modify how various agents perceive situations, weigh decisions, act and evaluate consequences.

Common wisdom is mostly concerned with harmonising the intentions, goals, objectives, constraints, commitments and plans of all the agents involved in the operation of a system. In short, social knowledge is based on the accumulated experience about handling conflicts. This knowledge appears at it best in the course of negotiations when disputants make their personal knowledge partly available to others; when they reveal step by step how far they are willing to go in sacrificing their own goals and exploiting their own resources for the sake of some (supposedly) common interest. Disputants create also new points of view through the dialogue and discussion. Conflict and disagreement pushes them to question explicit premises, even their own ones. During negotiations they learn to guess each others' intentions, constraints, co-operativeness, and reliability. 
Summing up, conflicts are and will be inherent components of manufacturing systems, as they are, for example, those of market economy (even market economy would not exist without conflicts that generate, after all, very efficient patterns of adaptive behaviour). They can give, however, discernible patterns and stability to social knowledge (c.f., Gasser, 1993). Accessing this knowledge as well as providing ways for using and enriching it with ever-changing individual experience are keys to integrating and co-ordinating the activities of various manufacturing agents.

\section{MODELING CONFLICTS IN MANUFACTURING SYSTEMS}

When dealing with conflicts, our aim was to develop a model that has an economy of description, holds the promise of the ease of use, but has, against all simplifications and abstractions, the power to suggest implications that are not evident at first sight. Our preliminary modelling assumptions concerning the environment in which conflicts may happen are as follows:

- The manufacturing system is modelled in a multi-agent framework: it is regarded as a network of agents that are able to communicate with each other. Some hierarchical organisational structure, however, can overlay on the network.

- The agents have only a partial (and, in a sense, distorted) model of the whole system: they may access a limited set of means for the acquisition and integration of new knowledge into their models and for pushing the system's state towards their own goals.

- The agents' knowledge referring to the same things is not necessarily commensurate, and may have also different representations.

- The overall system has a social character: its has properties which cannot be derived from the properties of its components.

- No closed-system assumption is maintained: the manufacturing system has to be modelled as being submerged into and interacting with the outside world that is not described by formal means. Since novel kinds of interaction with the world may occur, the model should be open and able to evolve.

Agents that may get in conflict with each other can be defined on several levels of abstraction. On the highest level, there are two parties only: Management and Production. In fact, many typical problems manifest themselves already on this level (see e.g., Shapiro, 1977). On more detailed levels there are agents like Product Design, Development, Process and Production Planning, Manufacturing, Marketing, Sales, etc. 
Management

Market share diminishes due to high relative price caused by gaps in the product line.

Objective

Action proposed

Objections against the other proposal

Difficulties with selling.
Production

Workload of the system gets lower than usual, inventory level of end products increases.

Decrease production cost.

Increase production volume by focusing the product line so as to be able to exploit economics of scale.

Delays in schedules and delivery. ${ }^{2}$

Figure 1: Example of a conflict situation.

1. Higher market share enables more control over distribution and customer; this, in turn, allows more effective scheduling of production, so overhead costs can be decreased. Hence, the high relative price may be decreased.

2. Broadening the product line needs investments in production equipment, moreover, its advantages can be seen after considerable delay. Broader product line results in higher variability of processing times.

The conceptual skeleton of conflicts is as follows: all parties assess the situation at hand, select their goals, objectives and relevant constraints and then propose an action. This action may be-due to differences in situation assessment or in the objective of the agents-unacceptable for the other parties. (As an example: the critical level of loading a capacity is different if seen with respect to near-term cash-flow versus long-term maintenance.) Now, supposing the parties have a mutual agreement in the overall objective of the system, they unravel their true objections against the other parties' proposals.

We have analysed various sources of conflicts in (Horváth et al., 1993). From among the several examples given there we show here only one typical case about a disagreement between Management and Production: a conflict of broadening versus focusing the product line (see Figure 1). 


\section{COOPERATIVE PROBLEM FORMATION AND SOLVING}

\subsection{Basic concepts}

Our vocabulary used for the description of the problem solving mechanism is as follows:

- Agents are who

- face the problems and make situation assessment;

- are responsible for solving the problems within their own authority;

- report the problems upward in the decision hierarchy of the system;

- initiate co-operative problem solving and participate in it;

- give advice in solving problems;

- execute directives received from the upper level of the decision hierarchy of the system;

- have a hidden inner structure and problem solving mechanism.

- Problems are

- any phenomena that need reaction other than prescribed by definitive operation rules;

- (re)formulated by the agents;

- solved either by direct action or after consultation or negotiation.

- Actions are made by agents for

- changing the system's state;

- passing directives and reports;

- serving other communicative purposes.

\subsection{The method of co-operative problem solving}

The method we suggest for co-operative problem solving supports both negotiation and mediation. The responsibility of solving a given control problem is always assigned to a particular agent. This agent in charge can then decide on whether to pass the responsibility, or to solve the problem. Problems are considered one after the other. Solution for a perceived problem is developed either by

- an immediate decision of the agent in charge;

- after a consultation with some invited agents, or

- after negotiation with some selected agents.

It is up to the agent in charge whether it passes responsibility, decides by its own, consults or negotiates with other agents. It is also its own right to select the partners; its communication channels may cross the boundaries of the organisational hierarchy. Conversely, when invited, agents may take part in the 
formulation and solution process of any problem, independently from their place in the hierarchy. However, it has to be emphasised that a poor decision (either due to lack of information and knowledge, or to the inappropriate selection of partners) may lead to a new problem situation at a higher decision level.

Final decision must be made by whomever in charge. This, and the decision and reporting hierarchy helps only to overcome secondary conflicts that ensue from the organisation structure alone (but not from the agents' disagreement over an issue).

In situations when the agents cannot fully describe their positions to another, yet they must mutually take some things for granted to act jointly, they enter a negotiation process. Negotiation is mediated-initiated, lead and concluded-by the agent in charge. Resolution is to be found in the course of this process through requalifying the parties' goal priorities and constraints, and leading to actions that are mutually acceptable and promise to reach the common objective.

In the negotiation process, the so-called conflict-base has a distinguished role. It is a store of generalised past conflict cases the overall system had faced during its operation. This memory embodies the collective knowledge of the agents operating in an ensemble. Whenever negotiation between conflicting agents leads to a deadend, the mediator turns to this memory and after retrieving similar problems it tries to re-use the past solutions under the actual circumstances. A very important aspect is that the conflict-base is continually evolving: new problem cases are learned while memories of old or irrelevant cases fade out.

The mechanism does not need to do automatic problem solving; instead, it should be the core of an advisory system that helps human parties approach a higher standard of mutual understanding via clarifying their conflicts and the possible resolutions.

\subsection{The protocol}

Here, we give a mechanism that enables the formation and solving of problems within the framework outlined above (see Figure 2). The mechanism leads through phases of distinct activities (reporting, initiating consultation, passing responsibility, etc.). It includes also a simple negotiation protocol.

Phases of problem formation and solving are described as schemata that should be interpreted in the following way: The frame-like structures on the left are intended to be filled in with formatted or free-form information, while the second column is for comments on the attributes. Phases of activities follow each other in the given order; alternative branches are denoted with letters.

Phase 1: Agent reports emerging problem

The emerging problem may be caused either by some internal disturbance of the system (e.g., machine breakdown), or an unexpected change in the external conditions (e.g., rush order, fall-out of a competitor). 


\begin{tabular}{|l|l|}
\hline Reporting agent & its identifier \\
\hline Factual description of the situation & relevant-looking facts \\
\hline Remarks & $\begin{array}{l}\text { initiating agent has a special right to comment } \\
\text { the situation, e.g., suggest an objective }\end{array}$ \\
\hline Deadline for response & \\
\hline Agent in charge & addressee of this report \\
\hline
\end{tabular}

Phase 2: Agent in charge is assigned

The agent who will be responsible for the solution of the problem from now on is assigned. Often it is the reporting agent itself who perceived the problem situation.

Phase 3A: Agent in charge acts or issues directive(s) to subordinate(s)

After having alone decided what to do, agent in charge acts directly or issues directive(s). There may be given several directives to different agents.

\begin{tabular}{|l|l|}
\hline Agent in charge & its identifier \\
\hline Directives & \\
\hline Agent addressed & \\
\hline Action required & \\
\hline Report required & what kind, to which agent(s) \\
\hline
\end{tabular}

Phase 3B: Agent in charge passes responsibility

Responsibility can be passed either on its own level or upward in the decision hierarchy. A new agent in charge will be assigned.

Phase 3C: Agent in charge initiates consultation

If there is no direct solutions (3A), and responsibility can neither be passed (3B), then the agent in charge has to organise a concerted action by inviting some other agents for consultation. In its own situation assessment the agent in charge outlines what goals to achieve, which objectives to approach and what constraints to satisfy.

\begin{tabular}{|l|l|}
\hline Agent in charge & its identifier \\
\hline Situation assessment & \\
\hline \multicolumn{1}{|c|}{ Goals and objectives } & \\
\hline \multicolumn{1}{|c|}{ Constraints } & factors to adhere \\
\hline Relaxable constraints & factors free to tune \\
\hline Negotiation protocol proposed & whether consultants may abstain, etc. \\
\hline Consulting agents called & \\
\hline
\end{tabular}

Phase 4: Evaluation of proposals

Consultants work out proposals, which are collected and evaluated by the agent in charge. Proposals are sent according to the following schema: 


\begin{tabular}{|l|l|}
\hline Consulting agent & its identifier \\
\hline Differences in situation assessment & $\begin{array}{l}\text { as related to that made by the agent in } \\
\text { charge }\end{array}$ \\
\hline Goals and objectives & \\
\hline Irrelevant constraints & \\
\hline Modified constraints & \\
\hline Additional constraints & new factors to consider \\
\hline Action proposed & \\
\hline Agent addressed & who shall execute action \\
\hline Description & \\
\hline Reporting & \\
\hline
\end{tabular}

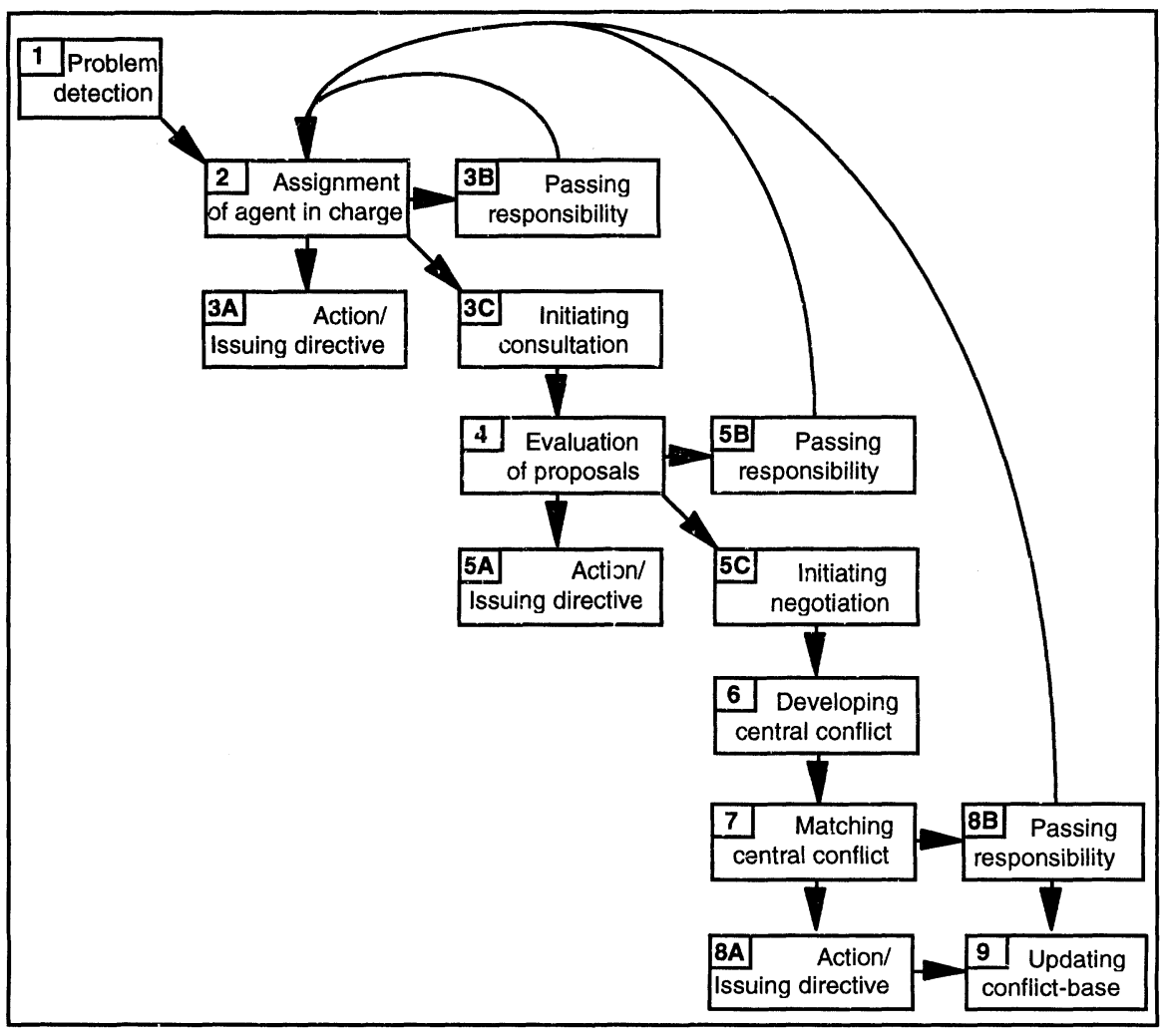

Figure 2: The problem formulation and solution process.

Phase 5A: Agent in charge accepts one proposal and issues directive(s)

If one of the proposals is totally acceptable for the agent in charge, it may act similar to Phase $3 \mathrm{~A}$. 
Phase 5B: Agent in charge passes responsibility upwards

If all proposals are totally unacceptable for the agent in charge, it may act similar to Phase 3B. However, the passing of responsibility within its own level would mean discarding the results of the consultation, so it is not allowed.

Phase 5C: Agent in charge initiates and moderates the negotiation over the proposals

In this phase a sequence of negotiation steps should lead to a better mutual understanding of the consultants' assessment of the situation, objective and agreement on action.

Step 5C.1: Broadcasting of proposals

Negotiating agents may ask for eventual clarification from each other.

Step 5C.2: Exposition of supporting and opposing relations of the negotiators

In this step, each agent invited to negotiate may support or oppose each other's proposal. There may be organised one or more cycles of mutual evaluation.

\begin{tabular}{|l|l|}
\hline Proposing agent & who presented the proposal \\
\hline Supporting/opposing agent & who relates itself to the proposal \\
\hline Differences & \\
\hline In situation assessment & \\
\hline In action & \\
\hline
\end{tabular}

Phase 6: Development of the central conflict in the situation

Supports are combined to yield proposals that will be considered as joint ones; however, these combinations neither provide a deeper insight into the problem nor suggest new paths toward its solution. On the other hand, conflicts exposed in the previous step may be more instructive since they show up the critical and ambiguous aspects of the current problem. Moreover, the comparison of problem cases, if sharply focused on these aspects, is more promising. During this comparison process, the agent in charge should found and/or develop a central conflict of the situation: 


\begin{tabular}{|c|c|}
\hline Statement of the central conflict & \\
\hline Side A: & covers several agents \\
\hline \multicolumn{2}{|l|}{ Situation assessment } \\
\hline \multicolumn{2}{|l|}{ Action proposed } \\
\hline Objection & to the proposed action of Side B \\
\hline \multicolumn{2}{|l|}{ Side B: } \\
\hline \multicolumn{2}{|l|}{ Situation assessment } \\
\hline \multicolumn{2}{|l|}{ Action proposed } \\
\hline Objection & to the proposed action of Side A \\
\hline $\begin{array}{l}\text { Characteristics of the resolution } \\
\text { process }\end{array}$ & $\begin{array}{l}\text { e.g., reconsider situation assessment by } \\
\text { accepting an alternative model or by re- } \\
\text { focusing time scale, reassign priorities of } \\
\text { objectives, propose another action }\end{array}$ \\
\hline
\end{tabular}

Phase 7: Matching the central conflict with the conflict-base

Now, the situation burdened by a conflict is matched via its characteristic features to the conflict-base: agent in charge looks for similar cases there.

\begin{tabular}{|c|l|}
\hline Central conflict of the situation & \\
\hline Conflict retrieved from conflict-base & one of several similar cases \\
\hline $\begin{array}{l}\text { Correspondence of past and } \\
\text { confrent }\end{array}$ & \\
\hline Degree of match & $\begin{array}{l}\text { consulting agents may have attached what } \\
\text { they had predicted would follow from the } \\
\text { decision }\end{array}$ \\
\hline Predicted outcomes & $\begin{array}{l}\text { the attribution of effects to previous problems } \\
\text { is questionable: the effects should be } \\
\text { indexed with the agents, too. }\end{array}$ \\
\hline
\end{tabular}

Phase 8A: Agent in charge accepts one proposal and issues directive(s)

After analysing the retrieved conflicts, the agent in charge decides to issue a directive. It acts like in Phase 3A.

Phase 8B: Agent in charge passes responsibility upwards

If matching of the conflict to the conflict-base does not help, then the agent in charge may act like in Phase 3B. Responsibility can be passed upward, together with the record of the negotiation process.

Phase 9: Updating the conflict-base

Consultants are informed about the conflict as crystallised in Phase 6 and about the final decision of the agent in charge. They are asked to send their last-word messages to predict consequences of the action. The annotated conflict is added to the conflict-base. 
With Phase 9 the processing of the problem terminates, and the co-operating system waits for a new problem.

\section{REASONING OVER CONFLICT CASES}

During the resolution of conflicts what accumulates over time is the knowledge of how to serve the community with one's own efforts without giving up self-identity. This knowledge, though it seems to belong to the individual, is relevant and valid only together with the background social knowledge of the other parties'; so it rather belongs to the organisation as a whole. This knowledge is tacit; i.e., it may happen that one possesses it but can not make it explicit even to herself. It is neither formal nor systematic, and is hard to communicate. It is not static; instead, it is attached strongly to a history and is continuously and dynamically evolving. It is hard to express in quantitative terms; e.g., as current cognitive investigations suggest, managers organise their memories about problems, conflict situations and resolutions in form of stories containing settings, characters, and plots (Goldstein, 1993, Hoch and Schkade, 1996).

The above features of knowledge about conflicts lead us to the conclusion that case-based reasoning (CBR) is the knowledge-transfer vehicle that can help us best in grasping and exploiting most pieces of knowledge that is related in some or other way to conflicts in manufacturing systems.

CBR is based on remembering past experiences when solving new problems (Kolodner, 1993, Leake, 1996). Cases are episodic descriptions of problems, their associated solutions and traces of the solution process (e.g., explanations). Retrieval of cases is governed by some kind of conceptual similarity. Beyond recalling, ideally it can perform the adaptation of previous cases, the validation of suggested solutions, and the recording of new cases. The merits of CBR as compared to other knowledge representation and reasoning techniques are that it (1) șupports reasoning over incomplete and incorrect data, (2) uses a representation that integrates problem statement, solution and its context, (3) allows direct incorporation of free-form textual information, (4) incorporates incremental learning, and (5) uses a human-readable knowledge-base. Hence, CBR is thought to be suitable for ill-structured domains that are difficult to model with a high level of correspondence. Thanks to the last three characteristics, it makes knowledge acquisition much easier to do.

In the course of the negotiation process, case-based reasoning can be of much help in a number of different roles, particularly in:

- assessing situation and clarifying, re-formulating problems;

- searching for, evaluating, and exploiting possible solution alternatives;

- projecting the outcome of decisions and performing what-if analysis;

- explaining situations and decisions;

- exercising critique by producing counter-examples; 
- learning and triggering further knowledge acquisition through collecting and generalising individual cases.

A case-based representation and reasoning scheme aimed at implementing the above functions must support, first of all, the retrieval and adaptation of the various compromises of the disputants in similar conflict situations. Hence, the case representation should include episodes characterised by problem setting, disputants and their conflict, trace of the negotiation process, accepted compromise, and actual outcome as evaluated by the disputants. Problem setting and the actual outcome of decision should refer to the flow of production; to the demand flow, loading, product mix, average capacity utilisation, schedule deviance, inventory level, mean lead times, total work-in-process, total output, direct operation costs, etc. Access to cases should be provided by indexing over salient features whose value may range from free-form textual information to symbols and numbers. However, indices to similar situations with very different compromises and/or outcomes are also needed.

Arguments against case-based reasoning say that it is inherently conservative; that by repeatedly applying well-proven, canned solutions it blocks imagination and innovation (see e.g., Hoch and Schkade, 1996). However, without a capacity of remembering, we would be too prone to repeat our previous mistakes. CBR covers not only the re-use of the results and/or processes of earlier problem solving activities, but also the accumulation, filtering and (re)organisation of these experiences. One can only hope that learning and innovation are not mutually exclusive. Secondly, imagination means rather a capacity to see all sides of a subject and to weigh all possibilities, than mere fantasy. In order to facilitate this, in our framework CBR is embedded into a problem formation and solving mechanism that is just for presenting alternative views of the same phenomena. E.g., when used for criticising pending decisions (in the role of the devil's advocate), a conflict-based decision support system would help innovation.

\section{DISCUSSION AND DIRECTIONS OF FUTURE WORK}

Our preliminary assumptions concerning conflicts are not conform with those underlying most conflict models of decision making theory (Zeleny 1982). In our case there is no guarantee that the same decision making frame is perceived by all agents; moreover, the agents' options and preferences do change under the negotiating process. One notable exception is (Fang et al. 1993) who consider conflict resolution as an interactive process which is free of the above-mentioned constraints. We emphasise, however, the need to learn all what can be distilled from the history of interactions; from the previous disputes with the same partners. Reasoning from precedent cases had also an application in negotiation support: it was used in a two-person game for restructuring labour management problems 
(Sycara, 1991), and later on it was proposed as a means for supporting concurrent engineering (Sycara, 1994). CBR was also used for making individual knowledge available to others in a large organisation (even across hierarchical boundaries). This system supported not directly co-operation but the transfer of knowledge related to software quality control (Kitano et al., 1992, Shimazu et al., 1993).

Recently, several more or less complementary methodologies have been developed for enterprise modelling, such as CIMOSA, PERA, and GRAI (for a comparison, see Williams et al., 1994). They unify functional (what to do), behavioural (when and how to do), resource, information processing and organisational (who has to do) aspects of modelling. These approaches take the locus of decision authorities for granted and concern mostly the efficiency of information transfer; i.e., how to organise, retrieve, sort, filter, transmit, and display information for designated decision makers. These models cover how to execute the processes that integrate the components of the enterprise, but cannot help much in dealing with common notions such as self-interest, adverse incentive, disagreement, conflict, negotiation, or consensus. We think that a layer for handling such concepts should be laid upon any decision structure, however well let it be organised. As an example, (Ayel, 1995) describes a simple supervisory architecture for resolving over-constrained decision problems in a manufacturing system that were designed according to the GRAI methodology.

The issue of conflicts asserts itself sharply in the context of the extended enterprise (Browne et al., 1995) where a manufacturing system is considered not as an isolated entity, but as a member of a complex network. In the main, the suggested problem formulation and solution mechanism would be appropriate in such a setting, too.

There are, however, several shortcomings in the scheme as presented above. Most notably:

- it is unable to process mutual conflicts in a parallel fashion: the first conflict has to be finished before starting with the next one;

- the concept of causal chains of conflicts is missing;

- similarity and contrast of conflicts, their classification, generalisation, combination and adaptation are to be worked out in detail later;

- factors that influence conflicts (e.g., horizon of interaction, belief about the effect of the own action on the rest of the group's future behaviour, size of the group) have been out of scope of the investigation;

- there is no theoretical explanation of how co-operation arises-and why and how that behaviour can suddenly change;

- questions of stability and equilibrium have not been addressed.

Currently, we aim at developing an assistant system that would support-but by no means automate-the collaboration of people who are engaged in various activities (such as corporate management, financing, marketing, product design etc.). In this stage of system development, our primary goal is knowledge 
acquisition by watching over the shoulders of decision-makers. Conflict analysis is being applied as a kind of book-keeping technique for recording conflicts and other social interactions among decision makers, and keeping track of decisions together with their consequences. Over time, as the accumulated knowledge grows and gets more and more sophisticated, the assistant system would gradually take over the responsibility of conflict management from the individuals.

\section{ACKNOWLEDGMENT}

This work has been supported by grant T017442 of the National Research Foundation of Hungary (OTKA).

\section{REFERENCES}

Ayel, J. (1995). Supervising conflicts in production management. Int. J. of Computer Integrated Manufacturing 8(1), 54-63.

Browne, J., Sackett, P.J. and Wortmann, J.C. (1995). Future manufacturing systems-Towards the extended enterprise. Computers in Industry 25, 235-254.

Brynjolfsson, E. and Mendelson, H. (1993). Information systems and the organization of modern enterprise. Journal of Organizational Computing 3(3), 245-255.

Constantine, L.L. (1993). Work organization: Paradigms for project management and organization. Communications of the ACM 36(10), 35-43.

Dilts, D.M., Boyd, N.P. and Whorms, H.H. (1991) The evolution of control architectures for automated manufacturing systems. Journal of Manufacturing Systems, 10(1), 79-92.

Fang, L., Hipel, K.W. and Kilgour, D.M. (1993). Interactive Decision Making. John Wiley and Sons.

Gasser, L. (1991). Social conceptions of knowledge and action: DAI foundations and open systems semantics. Artificial Intelligence 47, 107-138.

Gasser, L. (1993). Social knowledge and social action: Heterogeneity in practice. Proc. of the IJCAI-93 Conference, Morgan Kaufmann, 751-757.

Goldstein, D.K. (1993). Computer-based organizational learning: The importance of managers' stories. Journal of Organizational Computing 3(4), 417-442.

Hatvany, J. (1984). Optimizing the chances of survival. Annals of the CIRP 33(1), 345-346.

Hatvany, J. (1985). Intelligence and cooperation in heterarchic manufacturing systems. Robotics and Computer-Integrated Manufacturing 2(2), 101-104.

Hoch, S.J. and Schkade, D.A. (1996). A psychological approach to decision support systems. Management Science 42(1), 51-64. 
Horváth, M., Váncza, J. and Márkus, A. (1993). Conflicts in manufacturing systems - a problem setting. Information Infrastructure Systems for Manufacturing, (eds. Yoshikawa, H. and Goossenaerts, J.), North-Holland, 265-279.

Hyman, R.B. (1993). Creative chaos in high-performance teams: An experience report. Communications of the ACM 36(10), 57-60.

Kitano, H. et al. (1992). Building large-scale and corporate-wide case-based systems: Integration of organizational and machine executable algorithms. Proc. of the AAAI-92 Conference, AAAI/MIT Press, 843-849.

Kolodner, J. (1993). Case Based Reasoning. Morgan Kaufmann.

Leake, B.D. (ed.) (1996), Case-Based Reasoning: Experiences, Lessons, and Future Directions, AAAI Press/MIT Press, Menlo Park, CA.

Minsky, M. (1985). The Society of Mind. Simon and Schuster, New York.

Nonaka, I. (1991). The knowledge-creating company. Harvard Business Review, Nov-Dec. 1991, 96-104.

Shapiro, B.P. (1977). Can marketing and manufacturing coexist? Harvard Business Review, Sept-Oct. 1977, 104-114.

Shaw, M.J. and Fox, M.S. (1993). Distributed artificial intelligence for group decision support. Decision Support Systems 9, 349-367.

Shimazu, H., Kitano, H. and Shibata, A. (1993). Retrieving cases from relational databases: Another stride towards corporate-wide case-base systems. Proc. of the IJCAI-93 Conference, Morgan Kaufmann, 909-914.

Sycara, K.T. (1991). Problem restructuring in negotiation. Management Science 37(10), 1248-1268.

Sycara, K.T. (1994). A computational model of negotiation in concurrent engineering. Proc. of the Concurrent Engineering: Research and Applications Conf., Pittsburgh, 1994, 1-10.

Williams, T.J. et al. (1994). Architectures for integrating manufacturing activities and enterprises. Computers in Industry 24, 111-139.

Zeleny, M. (1982). Multiple-Criteria Decision Making. McGraw-Hill.

\section{BIOGRAPHIES}

M. Horváth is professor, Head of Department at the Technical University Budapest. He received M.Sc. (Mech. Eng.) degree in 1959, Ph. D. in 1966, D. Sc. in 1984. His research areas are adaptive control, optimisation of cutting conditions, multi-axis machining, flexible automation and process planning. Having developed several generative and vario-generative CAPP systems in the last years, he turned to AI methods in design and planning.

A. Márkus works at the Computer and Automation Research Institute of the Hungarian Academy of Sciences. He took M.Sc. degree in Mathematics at the 
Eötvös University Budapest (1975), and Ph.D. in Mechanical Engineering at the Technical University Budapest (1986). His research interest is related to the application of $\mathrm{AI}$ techniques in engineering.

J. Váncza is a research associate at the Computer and Automation Research Institute of the Hungarian Academy of Sciences. He received his M.Sc. degree (Electr. Eng.) in 1984, and his Ph.D. (Mech. Eng.) in 1994. His research interest is mainly in planning and learning methods of artificial intelligence, and the application of such methods to design, process planning and production management problems in manufacturing. 